\title{
Some Randomness Experiments on TRIVIUM
}

\author{
Subhabrata Samajder and Palash Sarkar \\ Applied Statistics Unit \\ Indian Statistical Institute \\ 203, B.T.Road, Kolkata, India - 700108. \\ \{subhabrata_r,palash\}@isical.ac.in
}

\begin{abstract}
The first output bit of TRIVIUM can be considered to be a boolean function of 80 key and 80 IV variables. Choose $n(n \leq 30)$ of the key variables and set the other variables to constant values. This gives an $n$-variable boolean function. In this work, we experimentally find examples of such boolean functions which deviate from a uniform random $n$-variable boolean function in a statistically significant manner. This improves upon the previously reported experimental 'non-randomness' result using the cube testing methodology by Aumasson et al in 2009 for TRIVIUM restricted to 885 rounds. In contrast, we work with full TRIVIUM and instead of using the cube methodology we directly find the algebraic normal form of the restricted version of the first output bit of TRIVIUM. We note, however, that our work does not indicate any weakness of TRIVIUM. On the other hand, the kind of experiments that we conduct for TRIVIUM can also be conducted for other ciphers.
\end{abstract}

Keywords : stream ciphers, TRIVIUM, statistical test, non-randomness.

\section{Introduction}

TRIVIUM is a hardware oriented synchronous stream cipher that was submitted to the Profile II (hardware) of the eSTREAM competition by Christophe De Cannière and Bart Preneel [DCP]. TRIVIUM maintains an internal state of size 288 bits. The state is subdivided into 3 shift registers of sizes 93,84 and 111 bits each. It uses a simple quadratic state update function with 3 AND operations as the only non-linear operations per round. There are 1152 initialization rounds. During the key generation, at each step, the state is updated and a single key bit is produced. This key bit is the XOR of 6 state bits. Over the years TRIVIUM has received much attention from the research community due to its simple structure. However, there is still no known attack on full version of TRIVIUM which works better than exhaustive search.

To gain a better understanding of the full cipher, scaled-down variants, such as Bivium A and Bivium B [Rad06], have been studied. Both Bivium A and Bivium B use two shift registers as their internal state unlike TRIVIUM which uses three. The attacks on TRIVIUM can be broadly classified into two categories. The first type analyses the scaled-down variants (Bivium A and Bivium B [Rad06]) and tries to extrapolate their results to the full TRIVIUM. The second approach has been to study the reduced-round variants of the cipher, i.e., TRIVIUM with ' $r$ ' rounds of key initialization where $r \leq 1152$.

Early results on reduced-round variants of TRIVIUM can be found in [TK07] and [Vie07]. In [TK07], Turan et al used Matsui's linear cryptanalysis method to get a linear approximation with a bias $2^{-31}$ for 288 rounds of key initialization. Whereas in [Vie07], Vielhaber used an IV resynchronization attack with 
$2^{6}$ IV's to break 576 rounds of TRIVIUM. Englund et al in [EJT07], experimentally showed statistical weakness in the keystream of TRIVIUM when reduced to 736 rounds of key initialization. In [O'N07], O'Neal claimed that TRIVIUM with 1152 rounds of key initialization may not be secure and proposed that the initialization rounds for TRIVIUM should be increased to $4 \times 1152=4608$ rounds. Fischer et al in [FKM08] used a framework for chosen IV statistical distinguishing analysis of stream ciphers to extract few key bits of TRIVIUM when reduced to 672 rounds of key initializations.

The cube attack was proposed in [DS09] by Dinur et al and used to recover the key after 767 initialization rounds. The attack required $2^{45}$ bit operations and the authors showed that this can be further reduced to $2^{36}$ bit operations. In [ADMS09], Aumasson et al introduced a new class of attacks called cube testers and developed distinguishers for 790 rounds of TRIVIUM with $2^{30}$ complexity and were able to detect non-randomness over 885 rounds in $2^{27}$ complexity, improving on the original 767 -round cube attack.

Recently in [FV13], Fouque and Vannet increased the number of attacked initialization rounds by improving the time complexity of computing cube. They were able to find a key recovery attack requiring $2^{39}$ queries for 784 initialization rounds and were also able to provide another key recovery attack up to 799 rounds with a complexity of $2^{40}$ for queries and $2^{62}$ for the exhaustive search part. In their attack, they used the Moebius Transform to improve on the time taken in the pre-processing stage of cube attack.

Our Results: The motivation for our work is the discovery of non-randomness after 885 rounds of TRIVIUM reported in [ADMS09]. We briefly discuss this result. The input key and IV variables are divided into two groups called cube variables $(\mathrm{CV})$ and superpoly variables $(\mathrm{SV})$. Suppose $g\left(x_{1}, \ldots, x_{c}\right.$; $\left.y_{1}, \ldots, y_{s}\right)$ denotes the boolean function representing the first keystream bit of TRIVIUM. There are $c+s$ input variables, where $\mathrm{CV}=\left\{x_{1}, \ldots, x_{c}\right\}$ and $\mathrm{SV}=\left\{y_{1}, \ldots, y_{s}\right\}$. Then superpoly $s_{C V}$ of $g$ corresponding to a cube of size $c$ is defined as

$$
s_{C V}\left(y_{1}, y_{2}, \ldots, y_{s}\right)=\bigoplus_{\left(x_{1}, x_{2}, \ldots, x_{c}\right) \in \mathbb{F}_{2}^{c}} g\left(x_{1}, x_{2}, \ldots, x_{c} ; y_{1}, y_{2}, \ldots, y_{s}\right),
$$

which is an $s$-variable boolean function in the variables SV. The details about the non-randomness of 885 rounds of TRIVIUM reported in [ADMS09] is a bit sketchy. We try to provide some more details. A cube of size 27 of IV variables mentioned in [DS09] was considered. Set all other IV variables to 0. It was experimentally discovered that in the superpoly corresponding to this cube, the key variables 1,4 and 5 are neutral (i.e., changing their values does not affect the outcome of the polynomial). It is mentioned that the zero key was used which would imply that all key bits other than 1, 4 and 5 were set to zero. It was argued that the discovery of such a polynomial in the structure of TRIVIUM is an evidence of non-randomness. This claim is also well accepted in the literature.

In general terms the above example can be viewed as follows. Let $g\left(x_{1}, \ldots, x_{c} ; y_{1}, \ldots, y_{s}\right)$ be the first output bit of TRIVIUM (after 885 rounds). The authors discover a transformation $\Phi$ such that the key bits 1, 4 and 5 are neutral for the boolean function $\Phi(g)$. The transformation $\Phi$ consists of applying the cube, setting IV and the other key bits to 0 .

The above kind of experimentally discovered 'non-randomness' after 885 rounds reported in [ADMS09] forms the motivation for our work. We ask the question as to whether it is possible to experimentally discover some kind of 'non-randomness' in full TRIVIUM. As above, if $g$ denotes the boolean function representing the first output bit, our goal is to discover a transformatiion $\Psi$ such that the boolean function $\Psi(g)$ shows some statistically quantifiable deviation from a uniform random function. The $\Psi$ that we consider does not involve evaluating a cube. The function $g$ depends on 80 key and 80 IV variables. The transformation $\Psi$ consists of choosing $n$ key variables and setting the other key and IV variables to 
constant values. As a result $\Psi(g)$ is a boolean function on $n$ variables. We are able to experimentally obtain examples of $\Psi(g)$ whose deviation from a uniform boolean function is statistically significant. Here $n$ is a parameter which is at most 30 .

The main computational challenge is to obtain the algebraic normal form (ANF) of $\Psi(g)$. For this we discuss two methods. The first one symbolically evolves TRIVIUM over the full 1152 rounds. This requires a fast algorithm for multiplying two boolean functions given by their ANFs and for this task we use the implementation reported in [Sam13]. The second method proceeds by first obtaining the truth table representation for $\Psi(g)$ and then using the Moebius transformation to obtain the ANF. Either of the methods yields both the ANF and also the truth table representation of $\Psi(g)$.

Suppose $u^{*}$ is a uniform random boolean function of $n$ variables. The weight of $u^{*}$ is a random variable with mean $2^{n-1}$. Given a probability $\alpha$, there is an interval $I_{\alpha}$ such that the weight of $u^{*}$ is in $I_{\alpha}$ with probability at least $\alpha$. We say that $\Psi(g)$ is unbalanced at level $\alpha$ if its weight lies outside the internal $I_{\alpha}$. Similar notions of algebraic unbalancedness for $\Psi(g)$ can be defined with respect to the total number of monomials in the ANF of $\Psi(g)$ and also with respect to the number of monomials of degree $d$ in the ANF of $\Psi(g)$. We also define a notion of unbalancedness over an $l$-dimensional uniform random vectorial boolean function $\tilde{u}^{*}$. Further details of the corresponding statistical tests are provided later.

In this work, we experimentally find concrete examples of $\Psi(g)$ for $n=10,20$ and 30 which are unbalanced, algebraically unbalanced with respect to the total number of monomials and also with respect to monomials of certain specific degrees. We further provide give concrete examples of $\Psi(\tilde{g})$ for $n=20,30$ (where $\tilde{g}$ denotes an $l$-dimensional vectorial boolean function) which are unbalanced. These results are obtained for level $\alpha$ corresponding to more than $99 \%$ probability. For lower values of $\alpha$, we are able to obtain examples of $\Psi(g)$ which simultaneously fail several of the statistical tests. Our experiments consist of randomly selecting the $n$ key variables and choosing the values for the other $160-n$ key and IV variables. This in effect randomly chooses the transformation $\Psi$. The reported results are obtained by randomly choosing possibilities for $\Psi$ several thousands of times.

We make no claims that our results exhibit a weakness of TRIVIUM. There are two implications of our work. First, our results show that to experimentally discover some 'non-random' polynomial in the structure of TRIVIUM, the complicated cube analysis technique of [ADMS09] is unnecessary. Instead one can simply look at the boolean function representing the first output bit by setting $160-n$ of the input variables to constant values. Second, our work discovers 'non-randomness' in TRIVIUM after the full 1152 rounds of initialization whereas [ADMS09] reported results only after 885 rounds.

The method described here is not particular to TRIVIUM. It can be applied to any cipher. Whether the results will be similar to that obtained for TRIVIUM is not clear and exploring this can form possible future work.

Related Work: We are not the first to consider applying statistical tests to the algebraic normal form of the output bits of a stream cipher. An early work by Filio [Fil02] and later follow-up in [Saa06] had explored this possibility. Our approach, however, differs from that of [Fil02, Saa06] in the following way. The work considered the ANFs of the first $N$ output bits of a stream cipher and investigated the distribution of monomials of degree $d$ in these ANFs for $d \leq 3$. The study was thus aggregated and unlike the specific 'non-randomness' example reported in [ADMS09]. 


\section{A Brief Description of TRIVIUM}

TRIVIUM maintains a 288-bit internal state "S" denoted by $S=\left(s_{1}, s_{2}, \ldots, s_{288}\right)$ and uses two algorithms, namely a key initialization algorithm, which we call the key and IV setup, and a key stream generation algorithm. The state $\mathrm{S}$ is further divided into 3 shift registers, namely $S_{1}=\left(s_{1}, s_{2}, \ldots, s_{93}\right), S_{2}=\left(s_{94}, s_{95}\right.$, $\left.\ldots, s_{177}\right)$ and $S_{3}=\left(s_{178}, s_{179}, \ldots, s_{288}\right)$.

\subsection{Key and IV Setup}

The algorithm is initialized by loading an 80 -bit key into the first 80 -bits of the state $\mathrm{S}$, i.e., $s_{1}, s_{2}, \ldots, s_{80}$ and an 80-bit IV into the state bits $s_{94}, s_{95}, \ldots, s_{173}$ and setting all remaining bits to 0 , except for $s_{286}, s_{287}$, and $s_{288}$, which are set to 1 . Each round of the iterative process extracts the values of 15 specific state bits and uses them to update 3 bits of the state. This is repeated for $4 \times 288=1152$ times. This can be summarized by the following pseudo-code (Algorithm 1):

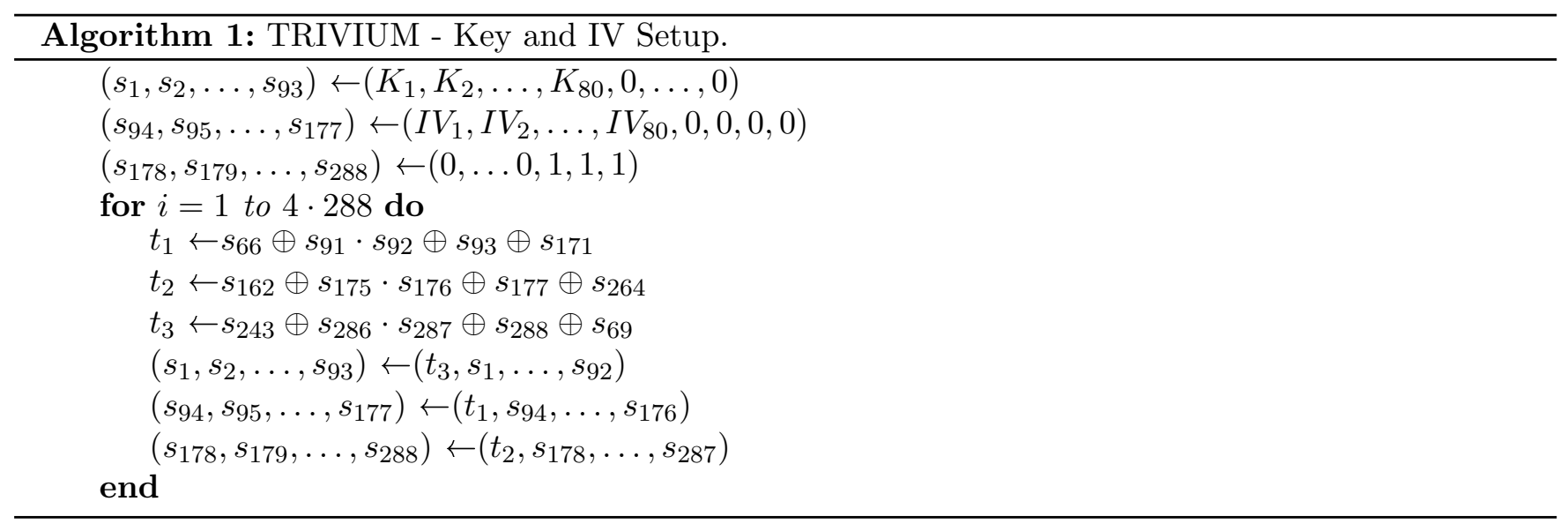

\subsection{Key Stream Generation}

The key stream generation algorithm is similar to that of the key initialization algorithm except that at each round, a single bit which is a linear function of six state bits, is output before the state update. This process repeats itself until the requested $N \leq 2^{64}$ bits of key stream is generated. The complete description is given by the following pseudo-code (Algorithm 2):

\section{Algebraic Normal Forms of the Output Bits of TRIVIUM}

Let us denote the key $K$ by $\left(k_{1}, k_{2}, \ldots, k_{80}\right)$ and the $I V$ by $\left(i v_{1}, i v_{2}, \ldots, i v_{80}\right)$. If instead of bits, we consider the key and the IV as variables then the state is initialized as follows:

$$
\begin{gathered}
\left(s_{1}, s_{2}, \ldots, s_{93}\right) \leftarrow\left(k_{1}, k_{2}, \ldots, k_{80}, 0, \ldots, 0\right), \\
\left(s_{94}, s_{95}, \ldots, s_{177}\right) \leftarrow\left(i v_{1}, i v_{2}, \ldots, i v_{80}, 0,0,0,0\right), \\
\left(s_{178}, s_{179}, \ldots, s_{288}\right) \leftarrow(0, \ldots 0,1,1,1) .
\end{gathered}
$$




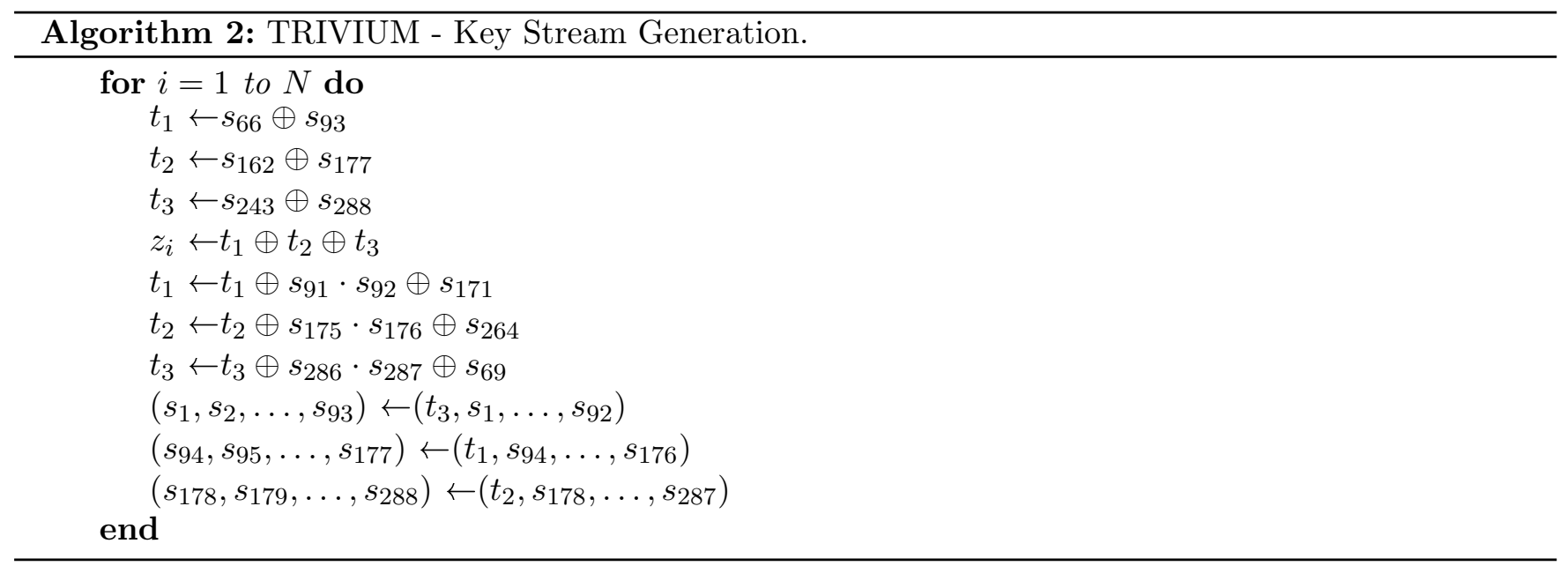

During each state update these state bits get multiplied and added in the boolean function ring defined over the variables $K$ and $I V$. Thus, considering each state bit as a boolean function in $80+80=160$ variables, one can view each state update as performing 3 multiplications $\left(1\right.$ for each $t_{i}, i=1,2,3$.) and 9 additions ( 3 for each $t_{i}, i=1,2,3$.). Addition is just bitwise XOR, whereas multiplication is that of two boolean functions given by their ANF's.

Handling the ANF of a boolean function on 160 variables is infeasible. Hence, we reduce the number of variables in the following manner. The key and IV bit positions which are to be treated as variables are randomly selected. These selected bit positions are then renamed as variables $k_{1}, k_{2}, \ldots, k_{n_{k}}$ and $i v_{1}, i v_{2}, \ldots, i v_{n_{i v}}$, such that $n_{k}+n_{i v}=n$. We work with $n \leq 30$. The rest of the key and IV bit positions are then set randomly to either 0 or 1 . The outputs bits of TRIVIUM can then be considered to be boolean functions of $n$ variables. We provide two methods to compute the ANFs of the output bits.

\subsection{Method - 1}

A symbolic computation of TRIVIUM is carried out where the state bits are treated as polynomials in $k_{1}, k_{2}, \ldots, k_{n_{k}}$ and $i v_{1}, i v_{2}, \ldots, i v_{n_{i v}}$. As a result, the first output bit which is the bitwise XOR of six state bits, namely $s_{66}, s_{93}, s_{162}, s_{177}, s_{243}$ and $s_{288}$ is also a polynomial in these variables.

The main time-consuming step in the above symbolic computation is that of multiplying the ANFs of

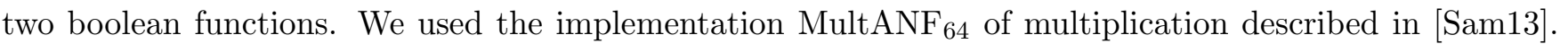
Using this algorithm, two 30-variable boolean functions can be multiplied in less than 2 seconds on a 3 GHz processor. Carrying out the simulation of full 1152 rounds of TRIVIUM with $n=30$ requires 3456 multiplications and the entire computation requires about one-and-half hours.

The complexity of Method - 1 is dominated by the number of multiplications of two $n$-variable boolean functions. We know from [Jou09] that the complexity for multiplying two $n$-variable boolean functions in their ANF is of the order of $3 n 2^{n}$. TRIVIUM uses 3 multiplications at each round. Therefore the total complexity of Method - 1 when used to evaluate an $r$-round variant of TRIVIUM (TRIVIUM with $r$ rounds of key initializations) is of the order of $9 r n 2^{n}$. 


\subsection{Method - 2}

The second method first constructs the truth table of the first output bit $z_{1}$ which is a polynomial in $n$ variables as mentioned above. A fast implementation of TRIVIUM is used to evaluate $z_{1}$ on all possible $2^{n}$ input combinations. This provides the truth table representation of $z_{1}$. This is converted into the ANF format using the Moebius transformation (see [Jou09] for a description of this algorithm).

The complexity of Method - 2 when used to evaluate $r$-round variant of TRIVIUM is $2^{n}$ computations of $r$-round TRIVIUM plus the cost required to convert the truth table of an $n$-variable boolean function into its corresponding ANF. And the cost for the conversion of the truth table to its ANF is $\mathcal{O}\left(n 2^{n}\right)$.

\subsection{Complexity of the Cube Tester}

Let the IV variables be divided into two parts the cube variables (CV) and the superpoly variables (SV). All the key variables are also treated as SV. Consider an $r$-round variant of TRIVIUM, i.e., TRIVIUM with $r$ rounds of key initialization. The cube tester [ADMS09] selects the cube variables from the 80 available IV variables. It treats the remaining key and IV variables as SV. It further divides the SV variables into two parts. Let us call the first part of variables as the function variables (FV) and retain the name SV for the second part of variables. In the cube testers for TRIVIUM mentioned in [ADMS09] the SV variables are all set to zero whereas the FV variables are set to fixed random values. Then $2^{C V}$ many executions of $r$-round TRIVIUM is used to find the value of the superpoly at ( $\mathrm{SV}, \mathrm{FV})$. This is repeated for $N$ different choices of FV. Therefore, to test one particular superpoly the attacker needs $N \times 2^{C V}$ executions of $r$-round TRIVIUM. In their paper [ADMS09] the authors had mainly concentrated on the cubes that were listed in the appendix of [DS09]. As mentioned in the paper the maximum size of FV and CV considered are 5 and 30 respectively. Therefore in order to compute the complete truth table of a 5 -variable function the cube tester has to compute $2^{35} r$-round variant of TRIVIUM.

\section{Some Elementary Statistics}

Let $X_{1}, \ldots, X_{n}$ be independent Bernoulli distributed random variables with $\operatorname{Pr}\left[X_{i}=0\right]=p$. Then $X=$ $X_{1}+\cdots+X_{N}$ follows $\operatorname{Bin}(N, p)$ with expected value $N p$. Given a probability $\alpha$, there is an interval $I_{\alpha}$ which is symmetric about the mean, such that $\operatorname{Pr}\left[X \in I_{\alpha}\right] \geq \alpha$. If $N$ is large enough, then the binomial distribution is well approximated by the normal distribution and it is quite routine to use the normal approximation to obtain $\operatorname{Pr}\left[X \in I_{\alpha}\right]$. Further, given $\alpha$, the interval $I_{\alpha}$ is found by converting to standard normal and then using tables for the standard normal distribution.

Denote by $u^{*}$ a uniform random $n$-variable polynomial. For our study, we will follow the above statistical approach for $u^{*}$ in the following settings.

Total number of monomials in $u^{*}$ : Any particular monomial occurs in $u^{*}$ with probability $1 / 2$ and is independent of the occurrence of any other monomial. If we denote the $2^{n}$ possible monomials of $n$ variables as $m_{0}, \ldots, m_{2^{n}-1}$, then we have $2^{n}$ random variables $X_{0}, \ldots, X_{2^{n}-1}$ where $X_{i}$ is 1 if $m_{i}$ is present in $u^{*}$ and 0 otherwise. The random variables $X_{0}, \ldots, X_{2^{n}-1}$ are independent Bernoulli distributed variables with $\operatorname{Pr}\left[X_{i}=1\right]=1 / 2$. The number of monomials in $u^{*}$ is $X=X_{0}+\cdots+X_{2^{n}-1}$ and follows $\operatorname{Bin}\left(2^{n}, 1 / 2\right)$.

Number of monomials of degree $d$ in $u^{*}$ : Consider the number of monomials of degree $d$ in $u^{*}$. There are a total of $\left(\begin{array}{l}n \\ d\end{array}\right)$ such monomials. In a manner similar to the above case, the number of monomials of 
degree $d$ in $u^{*}$ follows $\operatorname{Bin}\left(\left(\begin{array}{l}n \\ d\end{array}\right), 1 / 2\right)$.

Weight of $u^{*}$ : For any input, the output of $u^{*}$ is 0 or 1 with probability $1 / 2$ and this is independent of the output of $u^{*}$ on any other input. So, as in the case of total number of monomials, the weight of $u^{*}$ follows $\operatorname{Bin}\left(2^{n}, 1 / 2\right)$.

Determining whether a given polynomial is 'non-random': Given a particular $n$-variable boolean function $f$, we can compute the total number of monomials in $f$. If the number of monomials turns out to be 'significantly' away from $2^{n-1}$, then this is usually taken as an indication of some kind of non-randomness in $f$. We will use the term algebraically unbalanced to express the idea that the total number of monomials in $f$ deviates significantly from the expected number of monomials in a uniform random polynomial.

Statistical tests will be conducted as follows. For a probability $\alpha$, we first compute the interval $I_{\alpha}$ such that the total number of monomials in $u^{*}$ will be in $I_{\alpha}$ with probability at least $\alpha$. Then the total number of monomials in the given function $f$ is calculated. If this lies outside the interval $I_{\alpha}$, then we say that the function $f$ fails the algebraic balancedness test for probability $\alpha$, or that $f$ is algebraically unbalanced at level $\alpha$ written as $\mathrm{AU}_{\alpha}$.

In a similar manner we conduct tests on $f$ for monomials of degree $d$ and the weight of $f$. Given an $\alpha$, the interval $I_{\alpha}$ for the weight of $f$ will be the same as that for the total number of monomials. On the other hand, when considering monomials of degree $d$, the interval $I_{\alpha}^{(d)}$ will depend on $d$. This is because the number of trials in the binomial distribution corresponding to monomials of degree $d$ is $\left(\begin{array}{l}n \\ d\end{array}\right)$. If the number of monomials of degree $d$ in $f$ is outside the interval $I_{\alpha}^{(d)}$, then we will say that $f$ is $d$-algebraically unbalanced at level $\alpha$, written as $d$-AU $U_{\alpha}$. Similarly, if the weight of $f$ is outside the interval $I_{\alpha}$, then we say that $f$ is unbalanced at level $\alpha$, written as $\mathrm{U}_{\alpha}$.

\section{$5 \quad$ Unbalancedness Over First $l$ Output Bits}

This section generalizes the test for unbalancedness of a boolean function $f$ to vectorial boolean functions of dimension $l$. An $l$-dimensional vectorial boolean function is defined as $\tilde{f}: \mathbb{F}_{2}^{n} \rightarrow \mathbb{F}_{2}^{l}$, such that

$$
\tilde{f}\left(x_{1}, x_{2}, \ldots, x_{n}\right)=\left(f_{1}\left(x_{1}, x_{2}, \ldots, x_{n}\right), f_{2}\left(x_{1}, x_{2}, \ldots, x_{n}\right), \ldots, f_{l}\left(x_{1}, x_{2}, \ldots, x_{n}\right)\right)
$$

where each $f_{i}\left(x_{1}, x_{2}, \ldots, x_{n}\right), i=1,2, \ldots, l$ are $n$-variable boolean functions. Denote a uniform random vectorial boolean function by $\tilde{u}^{*}$, where each of its coordinates $u_{i}^{*}, i=1,2, \ldots, l$ behaves as uniformly and independently distributed $n$-variable polynomials.

Statistics Involved: Let $X_{i, j} \in\{0,1\}, i \in\{1,2,3, \ldots, l\}$ and $j \in\{1,2,3, \ldots, N\}$, denote mutually independent random variables with $X_{i, j} \sim \operatorname{Ber}\left(p_{i}\right)$ for all $j \in\{1,2, \ldots, N\}$. Let $X_{i}=\sum_{j=1}^{N} X_{i, j}$, $i \in\{1,2,3, \ldots, l\}$. Then $X_{1}, X_{2}, \ldots, X_{l}$ are independently distributed with each $X_{i} \sim \operatorname{Bin}\left(N, p_{i}\right)$. For sufficiently large $N, X_{i}$ 's are well approximated by normal distribution with mean $N p_{i}$ and variance $N p_{i}\left(1-p_{i}\right)$. Let $Y_{i}=\frac{X_{i}-N p_{i}}{\sqrt{N p_{i}\left(1-p_{i}\right)}}$. Then the random variables $Y_{1}, Y_{2}, \ldots, Y_{l}$ and hence $Y_{1}^{2}, Y_{2}^{2}, \ldots, Y_{l}^{2}$ are mutually independent with each $Y_{i} \sim \mathcal{N}(0,1)$ and $Y_{i}^{2} \sim \chi^{2}(1)$ (Chi-squared distribution with 1 degree of freedom). Therefore $\sum_{i=1}^{l} Y_{i}^{2} \sim \chi^{2}(l)$ (Chi-squared distribution with $l$ degree of freedom). For a given $\alpha$ and $l$ degrees of freedom, we therefore can get an interval $I_{\alpha}=\left[0, \chi^{2}(l)_{\alpha}\right]$ such that $\operatorname{Pr}\left[\sum_{i=1}^{l} Y_{i}^{2} \in I_{\alpha}\right]=\alpha$. 
Weight of $\tilde{u}^{*}$ : For any input and any coordinate $i(i \in\{1,2, \ldots, l\})$, the output of $u_{i}^{*}$ is 0 or 1 with probability $1 / 2$ and this is independent on any other input and coordinate $i$. For a given coordinate $i$ we denote the $2^{n}(=N)$ possible outputs of the $n$-variable boolean function $u_{i}^{*}$ as $X_{i, 0}, X_{i, 1}, \ldots, X_{i, 2^{n}-1}$, where $X_{i, j}$ is 1 if the $j^{\text {th }}$ output of $u_{i}^{*}$ is 0 and 0 otherwise. Given $i$, the random variables $X_{i, 0}, X_{i, 1}, \ldots, X_{i, 2^{n}-1}$ are independent Bernoulli distributed variables with $\operatorname{Pr}\left[X_{i, j}=1\right]=1 / 2$. Thus the number of zeros in the $2^{n}$ outputs of $u_{i}^{*}$ is $X_{i}=\sum_{j=0}^{2^{n}-1} X_{i, j}$ and follows $\operatorname{Bin}\left(2^{n}, 1 / 2\right)$. For $n \geq 5, X_{i}$ 's are well approximated by $\mathcal{N}\left(2^{n-1}, 2^{n-2}\right)$. Hence, the mutually independent random variables $Y_{i}^{2}=\left(\frac{X_{i}-2^{n-1}}{\sqrt{2^{n-2}}}\right)^{2} \sim \chi^{2}(1)$ for all $i \in\{1,2, \ldots, l\}$. Therefore, $\sum_{i=1}^{l} Y_{i}^{2} \sim \chi^{2}(l)$.

Determining a $l$-dimensional vectorial boolean function to be 'non-random': Given an $l$ dimensional vectorial boolean function $\tilde{f}$ in variables $x_{1}, x_{2}, \ldots, x_{n}$, we construct its $l$-dimensional truth table. For each of the $2^{n}$ possible values of the variables $x_{1}, x_{2}, \ldots, x_{n}$, we consider the corresponding values of $\tilde{f}\left(x_{1}, x_{2}, \ldots, x_{n}\right)$. This corresponds to the $l$-dimensional truth table of $\tilde{f}$. Each coordinate $i$ of this $l$-dimensional truth table individually corresponds to the truth table of $f_{i}$. Let $n_{i}$ denote the number of zeros in the truth table corresponding to $f_{i}$. Compute $\sum_{i=1}^{l}\left(\frac{n_{i}-2^{n-1}}{\sqrt{2^{n-2}}}\right)^{2}$ For a given $\alpha$ and $l$, if $\sum_{i=1}^{l}\left(\frac{n_{i}-2^{n-1}}{\sqrt{2^{n-2}}}\right)^{2}>\chi^{2}(l)_{\alpha}$ then we say that the $l$-dimensional vectorial boolean function $\tilde{f}$ is unbalanced at level $\alpha$, written as $\mathrm{U}_{l, \alpha}$

\section{Searching for (Algebraically) Unbalanced Polynomials}

As mentioned earlier, the first output bit of Trivium can be written as a boolean function of 80 key and $80 \mathrm{IV}$ variables. Since, it is infeasible to handle 160-variable boolean functions, we have used the following strategy to search for unbalanced polynomials.

1. Fix $n$ to be an integer which is at most 30 .

2. Out of the 80 key variables, choose $n$ key variables.

3. Set the remaining $80-n$ key variables and 80 IV variables to random binary values. This defines the first output bit to be a function $f$ of the $n$ key variables.

4. Use either Method-1 or Method-2 to obtain both the truth table representation and the algebraic normal form of the first output bit.

5. Determine whether $f$ is $\mathrm{AU}_{\alpha}, d-\mathrm{AU}_{\alpha}, \mathrm{U}_{\alpha}$ or $\mathrm{U}_{l, \alpha}$. For all the test except $\mathrm{U}_{l, \alpha}$ we have used 6 values $\alpha_{1}, \ldots, \alpha_{6}$ with $\alpha_{i}=1-1 / 2^{i+1}$ to conduct the tests. These values roughly correspond to $75 \%, 87.5 \%$, $93.75 \%, 96.88 \%, 98.44 \%$ and $99.22 \%$ probabilities respectively. For $\mathrm{U}_{l, \alpha}$ the value of $\alpha$ was set at $99.5 \%$.

Note that the above method randomly searches for a function $f$ which fails one or more of the tests. For a fixed $n$, Steps 2 and 3 above perform the task of selecting an $f$; Step 4 performs the task of generating the ANF of $f$; and finally Step 5 performs the test on $f$. If $f$ fails one or more of the tests, then this $f$ is reported.

The tests for different values of $\alpha$ are not independent. For $i>j, \alpha_{i}>\alpha_{j}$ and so, $I_{\alpha_{i}} \supset I_{\alpha_{j}}$. As a consequence, if a function $f$ is $\mathrm{AU}_{\alpha_{i}}$ then it is also $\mathrm{AU}_{\alpha_{j}}$. Similar comments hold for $d-\mathrm{AU}_{\alpha}$ and $\mathrm{U}_{\alpha}$. 


\subsection{Experimental Results}

The experiments were conducted by taking values of $n=10,20$ and 30 . Table 1 gives some polynomials for $n=10,20,30$, which are $\mathrm{U}_{\alpha_{6}}$, i.e., these polynomials are unbalanced at level $\alpha_{6}$. In the Table the column "Key Variables" indicate the key bit positions that were treated as variables. The columns "Key Constant" and "IV Constant" gives the values of $80-n$ and 80 bits of the key and IV bits which were set to constant values.

Consider once more what it means for a polynomial to be unbalanced at level $\alpha_{6}$. With probability $\alpha_{6}$, i.e., with about $99 \%$ probability, the weight of a uniform random function will be in the interval $I_{\alpha_{6}}$. Here we report examples of $f$ whose weight lies outside the interval $I_{\alpha_{6}}$. This indicates significant unbalancedness.

Table 1: Table showing list of polynomials which are $\mathrm{U}_{\alpha_{6}}$. The values given in the table are for $n=10,20,30$ and 1152 key initialization rounds of TRIVIUM.

\begin{tabular}{|c|c|c|c|c|}
\hline$n$ & Key Variables & Key Constant & IV Constant & $\begin{array}{l}\text { Number } \\
\text { of } 0 \text { 's }\end{array}$ \\
\hline \multirow{8}{*}{10} & \multirow{8}{*}{$1,4,22,38,42,44,53,56,61,78$} & OX452D5AA716418A9CC & OXBC925DE125682B159CB4 & 465 \\
\hline & & OX1476803AD7850AD36 & OXA1D62667224E6CF221CF & 465 \\
\hline & & OX31D5EC5914E3D922F & OXE24571405777B5521A & 555 \\
\hline & & OX54CD8D3B53FC0A114 & OXD4702BB150946D98D944 & 556 \\
\hline & & OX238009F $2 \mathrm{E} 69728 \mathrm{CB} 8$ & OX68131089DB607D1981F1 & 556 \\
\hline & & OX53DB1C63D36BB4FD2 & OXCF5050997F8601AB88EF & 558 \\
\hline & & OX42F216A6B2AFCEC17 & OX30E66D573F151F784B58 & 560 \\
\hline & & OX17485DC470A73061E & OXD54A1D5A59055062EFB6 & 571 \\
\hline \multirow{6}{*}{20} & $15,16,20,27,31,37,41,45,58,73$ & OX27F50AF693342B6F9 & OX706CCD7801037A0A49 & 437 \\
\hline & \multirow{5}{*}{$\begin{array}{c}0,1,9,10,14,19,27,29,41,42, \\
52,55,62,64,68,69,71,75,78,79\end{array}$} & OX3625E972822DB6A & OXB2D91DF4E87047E9B8C6 & 522657 \\
\hline & & OX80F5C4876AADE17 & OXA380363693475CFCCEB & 522768 \\
\hline & & OXB7521EE35C15C4B & OX309D70CFFD406A96299A & 522860 \\
\hline & & OXBCEFBB60D3A6BAF & OXB0EC6893275307067F03 & 522862 \\
\hline & & OXCD8AC4B29BEE0B1 & OX1DFF5B9FFE4363C2F1A3 & 522902 \\
\hline \multirow{3}{*}{30} & $\begin{array}{c}1,4,7,9,10,12,13,14,15,21, \\
25,27,30,31,32,33,34,44,52,54, \\
55,56,58,59,62,66,69,70,74,79\end{array}$ & OX290C10B0294D2 & OX586A33527C2928DDE2C6 & 536920658 \\
\hline & \multirow{2}{*}{$\begin{array}{l}7,15,20,21,22,26,29,30,32,33 \\
34,41,42,49,52,54,55,56,57,59 \\
60,63,64,65,66,72,75,76,78,79\end{array}$} & OX1FD41217D312F & OXC8C051B0D49C69D1A7DD & 536822130 \\
\hline & & OX12C5E491E4B6F & OX99E4748853D60D6617EC & 536920867 \\
\hline
\end{tabular}

Table 2, gives some polynomials for $n=10,20,30$, which are $\mathrm{AU}_{\alpha_{6}}$, i.e., these polynomials are algebraically unbalanced at level $\alpha_{6}$. Further, the entries $d$ in the column "Monomial Degrees" indicate that the corresponding function is also $d-\mathrm{AU}_{\alpha_{6}}$. The entry "None" in the column "Monomial Degrees" means that the corresponding function is not $d-\mathrm{AU}_{\alpha_{6}}$ for any value of $d=2,3, \ldots, n-2$. Again we note that the reported functions show algebraic unbalancedness at a level corresponding to $99.22 \%$ probability which indicates a significant deviation. 
Table 2: Table showing list of polynomials which are $\mathrm{AU}_{\alpha_{6}}$. Some of them are also $d-\mathrm{AU}_{\alpha_{6}}$. The values given in the table are for $n=10,20,30$ and 1152 key initialization rounds of TRIVIUM.

\begin{tabular}{|c|c|c|c|c|}
\hline$n$ & Key Variables & Key Constant & IV Constant & $\begin{array}{c}\text { Monomial } \\
\text { Degrees }\end{array}$ \\
\hline \multirow{5}{*}{10} & \multirow{4}{*}{$1,4,22,38,42,44,53,56,61,78$} & OX37FE4B0255D1D295C & OXD70079FAE0F0308EC206 & 6,8 \\
\hline & & OX457B6B0466DE7552E & OXD167CC3093E7E699466 & None \\
\hline & & OX0484EB9A3E80085D & OX9B10785F6BF67CA8D5CB & None \\
\hline & & OX243E3DFA82D00EE44 & OXB4526FDF61F96D7FCAE3 & None \\
\hline & $15,16,20,27,31,37,41,45,58,73$ & OX5EE252240CE406D5 & OX3F0E2249DE7C031CF797 & None \\
\hline \multirow{8}{*}{20} & \multirow{8}{*}{$\begin{array}{c}0,1,9,10,14,19,27,29,41,42, \\
52,55,62,64,68,69,71,75,78,79\end{array}$} & OX56B4A18579E0D3E & OXAC576EF0BDDE67E72619 & None \\
\hline & & OXFC12A46241151AD & OX10E6744E590F46973ADD & 13 \\
\hline & & OXADC520A5DA98587 & OX77EC7B17675B6489CAD8 & None \\
\hline & & OXC6AFA4B133A47F7 & OX61207A01BCC272B683F9 & None \\
\hline & & OX43ED55256B3CFF5 & OX822E158DE22B $7390747 \mathrm{~F}$ & None \\
\hline & & OXAA1BE 875 BC0B948 & OXE49D3F5E9DF3726567A & 10 \\
\hline & & OX44B684623514BE0 & OX9CB0767A4B911C07655B & 13 \\
\hline & & OXF9BB1A903D2B55A & OXBEFF617BF05E74ED8172 & 11 \\
\hline \multirow{4}{*}{30} & \multirow{4}{*}{$\begin{array}{l}7,15,20,21,22,26,29,30,32,33 \\
34,41,42,49,52,54,55,56,57,59 \\
60,63,64,65,66,72,75,76,78,79\end{array}$} & OXE65F $1294 \mathrm{C} 96 \mathrm{~A}$ & OXEB482AFBDFE04F8DAD56 & 14 \\
\hline & & OX128D80C2688E3 & OX3CF5643BE9AD30EAC0C8 & None \\
\hline & & OX199D831A8D833 & OX9F7651D0129823F00C61 & None \\
\hline & & OX1DBD945A6AD33 & OXDB855A93A2834AC2FE5C & 15 \\
\hline
\end{tabular}

Experiments to test $U_{l, \alpha}$ were done by taking values of $n=20,30$ and $l=8$. Tests were done by setting $\alpha=0.995$. Table 3 gives examples of polynomials which are $\mathrm{U}_{8,0.995}$ for $n=20,30$. For $n=20$, we found 4 such polynomials whereas for $n=30$ we found 6 polynomials.

Table 3: Table showing $\mathrm{U}_{8,0.995}$ polynomials for $n=20,30$.

\begin{tabular}{|c|c|c|c|}
\hline$n$ & Key Variables & Key Constant & IV Constant \\
\hline \multirow{4}{*}{20} & \multirow{4}{*}{$\begin{array}{c}0,4,10,11,18,19,20,21,29,30 \\
32,35,38,41,42,43,45,56,66,69\end{array}$} & OXE83EDFD172DA59E & OXD6985433DD11269B7EEC \\
\hline & & OX8976F5F031C8922 & OX7F8322315CFB $6675 \mathrm{E} 72 \mathrm{C}$ \\
\hline & & OX671FA8E37FA1559 & OX52CCAD8EF5C7C69766A \\
\hline & & OX25FB47658CE713C & OX73D27D4741280A814760 \\
\hline \multirow{6}{*}{30} & \multirow{4}{*}{$\begin{array}{c}0,2,3,4,7,8,12,19,23,26, \\
30,33,35,37,38,39,42,43,44,49 \\
54,58,60,62,63,64,65,70,72,76\end{array}$} & OX3646D112845B2 & OXB1E95646DCFA6FF10729 \\
\hline & & OX259294BDB83A1 & OX6028CA379F $720 \mathrm{ABC} 080$ \\
\hline & & OX11C4515398DDF & OXCEB11DDCCDCE6CD72BC1 \\
\hline & & OX188CF40F 48433 & OXE2F81539EA2F476236B3 \\
\hline & \multirow{2}{*}{$\begin{array}{c}0,1,5,6,7,12,13,14,21,24, \\
36,37,40,43,47,52,55,56,58,61 \\
63,64,65,68,72,73,75,77,78,79\end{array}$} & OX1067524FF3553 & OXD91F545A23C53ADC5796 \\
\hline & & $\mathrm{OX} 2588 \mathrm{D} 2 \mathrm{C} 38 \mathrm{E} 8 \mathrm{BF}$ & OX388A1E1866F8247F8D51 \\
\hline
\end{tabular}


We note that our experiments did not find any polynomial which simultaneously fail the tests for balancedness, total number of monomials and monomials of certain degrees at level $\alpha_{6}$. On the other hand, as we go down from level $\alpha_{6}$ to level $\alpha_{1}$, the experiments find more and more examples of polynomials simultaneously failing the tests for balancedness, total number of monomials and monomials of certain degree. Some examples are noted below and the details are given in the appendix.

1. Table 5 (Appendix A) gives a few polynomials which simultaneously fails the test for balancedness and the test for the total number of monomials at level $\alpha_{4}$. In addition, the table also gives the monomial degrees for which the test fails. We found 3 polynomials for $n=10$ and 2 polynomials for $n=30$ which had failed the test. However, we did not find any example for $n=20$.

2. Tables 4, 5, 6, 7, 8 (Appendix A) give examples of polynomials which simultaneously fail the three tests at levels $\alpha_{5}, \alpha_{4}, \alpha_{3}, \alpha_{2}$ and $\alpha_{1}$, respectively. In case of $\alpha=\alpha_{5}$, we can see from Table 4 that corresponding to $n=10$ we have only two polynomials which failed the tests, whereas we could not find any such examples for $n=20,30$. However, when the value of $\alpha$ was relaxed to $\alpha_{1}$, we found 45, 61 and 28 polynomials for $n=10,20$ and 30, respectively. The tables also show a steady increase in the number of monomials of a particular degree failing the test as $\alpha$ decreases.

\section{Conclusion}

In this paper, we have reported results of experiments conducted on the boolean function representing the first output bit of full TRIVIUM. These results show that by suitably restricting some of the input variables to constant values, it is possible to obtain polynomials which deviate from a uniform random polynomial in a statistically quantifiable manner. Our results may be considered as showing some kind of 'non-randomness' in full TRIVIUM. This is to be contrasted with the experimental evidence of 'nonrandomness' after 885 rounds reported in [ADMS09] using the complicated machinery of cube testers. We note on the one hand, that our results do not indicate any weakness in TRIVIUM, and on the other hand, that similar tests can be carried out on other ciphers.

At this point, we do not have any theoretical explanations for the experimentally obtained observations. Looking for such observations can form the task of future research.

\section{References}

[ADMS09] Jean-Philippe Aumasson, Itai Dinur, Willi Meier, and Adi Shamir. Cube Testers and Key Recovery Attacks on Reduced-Round MD6 and Trivium. In Fast Software Encryption. SpringerVarlag, 2009.

[DCP] Christophe De Cannière and Bart Preneel. Trivium-specifications. eSTREAM, ECRYPT Stream Cipher Project, Report 2005/030 (2005).

[DS09] Itai Dinur and Adi Shamir. Cube Attacks on Tweakable Black Box Polynomials. Advances in Cryptology-EUROCRYPT 2009, pages 278-299, 2009.

[EJT07] Håkan Englund, Thomas Johansson, and Mettem Sönmez Turan. A Framework for Chosen IV Statistical Analysis of Stream Ciphers. In INDOCRYPT, pages 268-281, 2007. 
[Fil02] Eric Filiol. A New Statistical Testing for Symmetric Ciphers and Hash Functions. In Information and Communications Security, pages 342-353. Springer, 2002.

[FKM08] Simon Fischer, Shahram Khazaei, and Willi Meier. Chosen IV Statistical Analysis for Key Recovery Attacks on Stream Ciphers. In AFRICACRYPT, pages 236-245, 2008.

[FV13] Pierre-Alain Fouque and Thomas Vannet. Improving Key Recovery to 784 and 799 rounds of Trivium using Optimized Cube Attacks. In Fast Software Encryption. Springer, 2013.

[Jou09] Antoine Joux. Algorithmic cryptanalysis. CRC Press, 2009.

[O'N07] Sean O'Neil. Algebraic Structure Defectoscopy. In Special ECRYPT Workshop-Tools for Cryptanalysis, 2007.

[Rad06] Håvard Raddum. Cryptanalytic Results on Trivium. Technical Report 2006/039, eSTREAM, ECRYPT Stream Cipher Project, Report, http://www.ecrypt.eu.org/stream/papersdir/2006/039.ps, 2006.

[Saa06] Markku-Juhani O Saarinen. Chosen-IV Statistical Attacks on eSTREAM Stream Ciphers. Proc. Stream Ciphers Revisited SASC, 2006.

[Sam13] Samajder, Subhabrata and Sarkar, Palash. Fast Multiplication of the Algebraic Normal Forms of Two Boolean Functions. In Lilya Budaghyan, Tor Helleseth and Matthew G. Parker, editor, WCC 2013, pages 373-385, 2013. (pdf available at http://www.selmer.uib.no/WCC2013/ pdfs/Samajder.pdf).

[TK07] M Sönmez Turan and Orhun Kara. Linear Approximations for 2-round TRIVIUM. In Proc. First International Conference on Security of Information and Networks (SIN 2007), pages 96-105, 2007.

[Vie07] M. Vielhaber. Breaking One.Fivium By AIDA : An Algebraic IV Differential Attack. Technical Report 2007/413, Cryptology ePrint Archive, Report, http://eprint.iacr.org/2007/413, 2007.

\section{A Tables}

Table 4: Table showing list of polynomials which are both $\mathrm{AU}_{\alpha_{5}}$ and $\mathrm{U}_{\alpha_{5}}$. Some of them are also $d-\mathrm{AU}_{\alpha_{5}}$. The values given in the table are for $n=10,20,30$ and 1152 key initialization rounds of TRIVIUM.

\begin{tabular}{|c|c|c|c|c|}
\hline$n$ & Key Variables & Key Constant & IV Constant & $\begin{array}{c}\text { Monomial } \\
\text { Degrees }\end{array}$ \\
\hline \hline \multirow{2}{*}{10} & \multirow{2}{*}{$1,4,22,38,42,44,53,56,61,78$} & OX37BDD3EAD0BAFABC0 & OXDB565D9DB98F4E3389C5 & None \\
\cline { 3 - 5 } & & OX47E214EB5727E04C9 & OXD34F684B1055DAECE93 & 7 \\
\hline
\end{tabular}


Table 5: Table showing list of polynomials which are both $\mathrm{AU}_{\alpha_{4}}$ and $\mathrm{U}_{\alpha_{4}}$. Some of them are also $d-\mathrm{AU}_{\alpha_{4}}$. The values given in the table are for $n=10,20,30$ and 1152 key initialization rounds of TRIVIUM.

\begin{tabular}{|c|c|c|c|c|}
\hline$n$ & Key Variables & Key Constant & IV Constant & $\begin{array}{c}\text { Monomial } \\
\text { Degrees }\end{array}$ \\
\hline \multirow{3}{*}{10} & \multirow{3}{*}{$1,4,22,38,42,44,53,56,61,78$} & OX37BDD3EAD0BAFABC0 & OXDB565D9DB98F4E3389C5 & None \\
\hline & & OX47E214EB5727E04C9 & OXD34F684B1055DAECE93 & 7 \\
\hline & & OX4547D85442C8D68CF & OXD08829A188F6241E7C2D & 5,8 \\
\hline \multirow{2}{*}{30} & \multirow{2}{*}{$\begin{array}{l}7,15,20,21,22,26,29,30,32,33, \\
34,41,42,49,52,54,55,56,57,59, \\
60,63,64,65,66,72,75,76,78,79\end{array}$} & OX11D3963CFE658 & OXE9F618EC66862A0DEB4E & 12 \\
\hline & & OX191766116C74F & OX1E8B7D71045E0F56A4EA & 4,24 \\
\hline
\end{tabular}

Table 6: Table showing list of polynomials which are both $\mathrm{AU}_{\alpha_{3}}$ and $\mathrm{U}_{\alpha_{3}}$. Some of them are also $d-\mathrm{AU}_{\alpha_{3}}$. The values given in the table are for $n=10,20,30$ and 1152 key initialization rounds of TRIVIUM.

\begin{tabular}{|c|c|c|c|c|}
\hline$n$ & Key Variables & Key Constant & IV Constant & $\begin{array}{c}\text { Monomial } \\
\text { Degrees }\end{array}$ \\
\hline \multirow{3}{*}{10} & \multirow{3}{*}{$1,4,22,38,42,44,53,56,61,78$} & OX37BDD3EAD0BAFABC0 & OXDB565D9DB98F4E3389C5 & None \\
\hline & & OX47E214EB5727E04C9 & OXD34F684B1055DAECE93 & 2,7 \\
\hline & & OX4547D85442C8D68CF & OXD08829A188F6241E7C2D & 5,8 \\
\hline \multirow{2}{*}{20} & \multirow{2}{*}{$\begin{array}{c}0,1,9,10,14,19,27,29,41,42 \\
52,55,62,64,68,69,71,75,78,79\end{array}$} & OX6725535534737CA & OXDDAE21B901422A1643A & None \\
\hline & & OXDDAE21B901422A1643A & OXF51C70E932C18D17D41 & None \\
\hline \multirow{4}{*}{30} & \multirow{4}{*}{$\begin{array}{c}7,15,20,21,22,26,29,30,32,33 \\
34,41,42,49,52,54,55,56,57,59 \\
60,63,64,65,66,72,75,76,78,79\end{array}$} & OX11D3963CFE658 & OXE9F618EC66862A0DEB4E & 12 \\
\hline & & OX3C25D092EFEF9 & OXEE0C5AA49BA676F04E05 & 14,15 \\
\hline & & OX613242AFA99E & OX74996C308B57426EC1FF & 4,13 \\
\hline & & OX191766116C74F & OX1E8B7D71045E0F56A4EA & 4,24 \\
\hline
\end{tabular}

Table 7: Table showing list of polynomials which are both $\mathrm{AU}_{\alpha_{2}}$ and $U_{\alpha_{2}}$. Some of them are also $d-\mathrm{AU}_{\alpha_{2}}$. The values given in the table are for $n=10,20,30$ and 1152 key initialization rounds of TRIVIUM.

\begin{tabular}{|c|c|c|c|c|}
\hline$n$ & Key Variables & Key Constant & IV Constant & $\begin{array}{c}\text { Monomial } \\
\text { Degrees }\end{array}$ \\
\hline \multirow{10}{*}{10} & \multirow{10}{*}{$1,4,22,38,42,44,53,56,61,78$} & OX37BDD3EAD0BAFABC0 & OXDB565D9DB98F4E3389C5 & 3,4 \\
\hline & & OX47E214EB5727E04C9 & OXD34F684B1055DAECE93 & 2,7 \\
\hline & & OX4547D85442C8D68CF & OXD08829A188F6241E7C2D & $4,5,8$ \\
\hline & & OX22C $82 \mathrm{FA} 5 \mathrm{C} 4 \mathrm{FF} 1 \mathrm{FFA} 7$ & OX8E6C7CCC6DA42DE02582 & 6 \\
\hline & & OX $46 \mathrm{~F} 73734324 \mathrm{~A} 8 \mathrm{C} 3 \mathrm{CF}$ & OXED6F602BFE6161C4B002 & $5,7,8$ \\
\hline & & OX548E1A39B23F 3483B & OX2C2B1447188F2DF15053 & 6,8 \\
\hline & & OX52A20EB6B6861FD2B & OX69EF 224FC6FB72AC6C37 & 2,3 \\
\hline & & OX243E3DFA82D00EE44 & OXB4526FDF61F96D7FCAE3 & 5,6 \\
\hline & & OX21FEF73EB0DC5739A & OX7598278A31B96B6E06F5 & 3 \\
\hline & & OX36B1281D43A9240B3 & OXE58A191A1E6C333C8EFD & 3,5 \\
\hline
\end{tabular}

Continued on next page 
Table 7 - Continued from previous page

\begin{tabular}{|c|c|c|c|c|}
\hline$n$ & Key Variables & Key Constant & IV Constant & $\begin{array}{l}\text { Monomial } \\
\text { Degrees }\end{array}$ \\
\hline \multirow{2}{*}{10} & \multirow{2}{*}{$1,4,22,38,42,44,53,56,61,78$} & OX1185DD59742FE8169 & OX89B62A60C21C42A0E6B2 & 4 , \\
\hline & & OX37FE4B0255D1D295C & OXD70079FAE0F0308EC206 & $6,7,8$ \\
\hline \multirow{16}{*}{20} & \multirow{16}{*}{$\begin{array}{c}0,1,9,10,14,19,27,29,41,42, \\
52,55,62,64,68,69,71,75,78,79\end{array}$} & OX6725535534737CA & OXDDAE21B901422A1643A & $8,12,15,17$ \\
\hline & & OXC481FD7BC1F523 & OXC37057969DFB005C79DC & $5,6,8,9$ \\
\hline & & OXDF7305B0CFDB228 & OXC9C17DF5198908297669 & 8 \\
\hline & & OX3480FFC0AD084D6 & OXF51C70E932C18D17D41 & 9,15 \\
\hline & & OX80A26F93FFE786E & OX49025F652E977970AAA3 & $3,5,6,9$ \\
\hline & & OX99997304FBA97AB & OX91A0123D835369D66539 & $3,10,11$ \\
\hline & & OX1438B4C6E410610 & OXEC881E225AE17BE12D06 & 6,16 \\
\hline & & OX2B8619E6B23FD69 & OXF24C75F66F5957352674 & None \\
\hline & & OX2E93E577A837AAC & OXF50C2B06C5B100F1D712 & 2,14 \\
\hline & & OXFDAFFE872B1ECA6 & OX63F0791A5BD92EA49167 & $2,7,10$ \\
\hline & & OX1E15EFE0723A1A0 & OXAFF45320480C32FE05AD & $11,12,13,14,17$ \\
\hline & & OX94252897FEBA & OX40B53BED60BA2A4EF7BD & 7 \\
\hline & & OX5A4644E0DCF37F1 & OXAFE71BE0360E0C918B9C & $3,9,13$ \\
\hline & & OXAA07D7C6F 262C91 & OX4F821468B1891D2AD371 & $\begin{array}{l}2,3,4 \\
12\end{array}$ \\
\hline & & OX748AA0B4C4431F6 & OX6BB3415153E252D74428 & 9 \\
\hline & & OX82641E96DDFE210 & OXA045545ADF 754FE 49440 & $4,16,17$ \\
\hline \multirow{5}{*}{30} & \multirow{5}{*}{$\begin{array}{c}7,15,20,21,22,26,29,30,32,33 \\
34,41,42,49,52,54,55,56,57,59 \\
60,63,64,65,66,72,75,76,78,79\end{array}$} & OX11D3963CFE658 & OXE9F618EC66862A0DEB4E & $12,15,18$ \\
\hline & & OX3C25D092EFEF9 & OXEE0C5AA49BA676F04E05 & $14,15,23$ \\
\hline & & OXCDCA70B4903D & OX28094F93A84519B6030 & $\begin{array}{l}2,12,21 \\
26\end{array}$ \\
\hline & & OX613242AFA99E & OX74996C308B57426EC1FF & $4,13,15,19,24,26,27$ \\
\hline & & OX191766116C74F & OX1E8B7D71045E0F56A4EA & $4,18,24,25$ \\
\hline
\end{tabular}

Table 8: Table showing list of polynomials which are both $\mathrm{AU}_{\alpha_{1}}$ and $\mathrm{U}_{\alpha_{1}}$. Some of them are also $d-\mathrm{AU}_{\alpha_{1}}$. The values given in the table are for $n=10,20,30$ and 1152 key initialization rounds of TRIVIUM.

\begin{tabular}{|c|c|c|c|c|}
\hline$n$ & Key Variables & Key Constant & IV Constant & $\begin{array}{c}\text { Monomial } \\
\text { Degrees }\end{array}$ \\
\hline \multirow{10}{*}{10} & \multirow{10}{*}{$1,4,22,38,42,44,53,56,61,78$} & OX37BDD3EAD0BAFABC0 & OXDB565D9DB98F4E3389C5 & $2,3,4,5$ \\
\hline & & OX22C 82 FA 54 FF $1 F F A 7$ & OX8E6C7CCC6DA42DE02582 & 6 \\
\hline & & OX47E214EB5727E04C9 & OXD34F684B1055DAECE93 & $2,4,7$ \\
\hline & & OX4547D85442C8D68CF & OXD08829A188F6241E7C2D & $4,5,8$ \\
\hline & & OX46F 73734324A8C3CF & OXED6F602BFE6161C4B002 & $5,7,8$ \\
\hline & & OX548E1A39B23F3483B & OX2C2B1447188F2DF15053 & $6,7,8$ \\
\hline & & OX25999FA70096CE14A & OX534E7B0E0099371CEC2B & 7 \\
\hline & & OX32C3B8564711127E2 & OXC3B65FA580064682A886 & $2,5,6,7,8$ \\
\hline & & OX36700C6F525F4A15E & OX76C0CA $72 \mathrm{E} 4037279 \mathrm{E} 52$ & $2,5,7$ \\
\hline & & OX1104F75E2114D99C6 & OX99545A9EDB9B664CF25E & 5,6 \\
\hline
\end{tabular}


Table 8 - Continued from previous page

\begin{tabular}{|c|c|c|c|c|}
\hline$n$ & Key Variables & Key Constant & IV Constant & $\begin{array}{c}\text { Monomial } \\
\text { Degrees }\end{array}$ \\
\hline \multirow{35}{*}{10} & \multirow{35}{*}{$1,4,22,38,42,44,53,56,61,78$} & OX52A20EB6B6861FD2B & OX69EF $224 \mathrm{FC} 6 \mathrm{FB} 72 \mathrm{AC} 6 \mathrm{C} 37$ & $2,3,6$ \\
\hline & & OX243E3DFA82D00EE44 & OXB4526FDF61F96D7FCAE3 & $5,6,7$ \\
\hline & & OX21FEF73EB0DC5739A & OX7598278A31B96B6E06F5 & 2,3 \\
\hline & & OX36B1281D43A9240B3 & OXE58A191A1E6C333C8EFD & $2,3,5$ \\
\hline & & OX373444186660E5FF 4 & OXEB195ADDBE903C2D1056 & 2 \\
\hline & & OX1185DD59742FE8169 & OX89B62A60C21C42A0E6B2 & 4 \\
\hline & & OX13C98DD112B9E4345 & OXC02E2FE44559226743EA & 2,6 \\
\hline & & OX25964FF8044895C95 & OXCFC776D1C4E100F 35C85 & $2,4,5$ \\
\hline & & OX2628BA81850F8F769 & OX1FCF571CE4612534B608 & 4,8 \\
\hline & & OX043DACA1A2026DBDA & OXBDC5DCD77F921AABDF6 & 4,7 \\
\hline & & OX2126745C279E5A10C & OX1248772E03E133CE0B7B & 2,7 \\
\hline & & OX586B4E14496FAC82 & OX9C2138672ABB3DDDC1F2 & 5 \\
\hline & & $\mathrm{OX} 368252990744 \mathrm{C} 0 \mathrm{C} 7$ & OX74DF32D819F351C27B0E & $2,3,4$ \\
\hline & & OX532D3BE97067D5129 & OX7BED67B3ABB91C35FA5D & 5 \\
\hline & & OX1382D64B413855656 & OX95972A312ED14A3BF60 & 6 \\
\hline & & OX52FFFC0FF6FD88EBD & OXF66572321FFA19728935 & 3,7 \\
\hline & & OX439C148480CEF 8257 & OX74FB1106EE59338B8704 & 3 \\
\hline & & OX14B0825030BA0A96B & OX207C5A11622E7FE89689 & $2,3,7$ \\
\hline & & OX568F9EDC3FA5CFC5 & OXCD8D6086AF815B848C24 & $3,6,8$ \\
\hline & & OX37A8A2D3F4AD45193 & OXD49B28D3ABC66F27C37E & 3,6 \\
\hline & & OX457B6B0466DE7552E & OXD167CC3093E7E699466 & 5,6 \\
\hline & & OX2CE451DE26F01574 & OX1C1342B3181C132E7CCF & 8 \\
\hline & & OX2188425AC63CCD33F & OX90C929DD67D3678472EE & $2,4,5,6,8$ \\
\hline & & OX2454FEF2819CFDFE8 & OX9E71576A5F36051743D5 & 6,7 \\
\hline & & OX04D00A4F9785AC8C4 & OX8DCA7A16BF435CE5940B & 5 \\
\hline & & OX37FE4B0255D1D295C & OXD70079FAE0F0308EC206 & $4,6,7,8$ \\
\hline & & OX53CD508F74BBC7DBE & $\mathrm{OXC} 37 \mathrm{E} 2 \mathrm{~A} 7 \mathrm{~F} 2 \mathrm{~F} 8164 \mathrm{D} 022 \mathrm{BB}$ & $5,7,8$ \\
\hline & & OX048DAFF69510A4B0E & OX3A1A19897D5D77691BDB & 5 \\
\hline & & OX4708A09334FCAFE4F & OX4A4C60F2FECB3B1FFA4F & $2,3,5$ \\
\hline & & OX4559C324D7A96E402 & OX76093CA6A6820F 188476 & 6 \\
\hline & & OX11CCD131147B71B01 & OX82C85493CE4525CC267A & 4,7 \\
\hline & & OX43C5AE65F 459310FF & OXE29F6C5FF9B122017D55 & 6 \\
\hline & & OX074D96A9360193375 & OXFA274E2AFE2E68F3ECE6 & 6 \\
\hline & & OX073A6C0377AF88B83 & OX6351165578DB3B77F014 & 3,5 \\
\hline & & OX11D7C2096469B8D59 & OX85164E66AB350C5BCD85 & 2 \\
\hline \multirow{6}{*}{20} & \multirow{6}{*}{$\begin{array}{c}0,1,9,10,14,19,27,29,41,42 \\
52,55,62,64,68,69,71,75,78,79\end{array}$} & OXCD8AC4B29BEE0B1 & OX1DFF5B9FFE4363C2F1A3 & $2,5,8,13,17$ \\
\hline & & OX6725535534737CA & OXDDAE21B901422A1643A & $8,12,15,16,17$ \\
\hline & & OXC481FD7BC1F523 & OXC37057969DFB005C79DC & $5,6,8,9,14$ \\
\hline & & OXDF7305B0CFDB228 & OXC9C17DF5198908297669 & $4,6,8,17,18$ \\
\hline & & OXE8EF47A657A1A10 & OX47B07462C84600BBD4C3 & $8,12,17$ \\
\hline & & OX3480FFC0AD084D6 & OXF51C70E932C18D17D41 & $4,6,9,14,15$ \\
\hline
\end{tabular}


Table 8 - Continued from previous page

\begin{tabular}{|c|c|c|c|c|}
\hline$n$ & Key Variables & Key Constant & IV Constant & $\begin{array}{c}\text { Monomial } \\
\text { Degrees }\end{array}$ \\
\hline \multirow{41}{*}{20} & \multirow{41}{*}{$\begin{array}{c}0,1,9,10,14,19,27,29,41,42 \\
52,55,62,64,68,69,71,75,78,79\end{array}$} & OXF79DC891384AFF0 & OXDDAF $2 \mathrm{C} 635 \mathrm{~F} 1725 \mathrm{E} 5722 \mathrm{C}$ & $2,3,5,11,15,17$ \\
\hline & & OX63CBCE13DFA0AFF & OXC314A7271C748FEB77B & $3,11,15$ \\
\hline & & OX80A26F93FFE786E & OX49025F652E977970AAA3 & $3,4,5,6,9,14$ \\
\hline & & OX99997304FBA97AB & OX91A0123D835369D66539 & $3,10,11$ \\
\hline & & OX1438B4C6E410610 & OXEC881E225AE17BE12D06 & $2,6,7,9,16$ \\
\hline & & OX2B8619E6B23FD69 & OXF24C75F 66F5957352674 & 10,13 \\
\hline & & OX3907E66406C1230 & OXCC4F643389D174E09308 & $9,11,12,17$ \\
\hline & & OX8DC9AD07E1DD5B7 & OX6F925A43AABE3589016F & $2,9,18$ \\
\hline & & OX1C2904C43FF6577 & OXE73A4739E4C117D70E8E & $7,11,12,13,16,17$ \\
\hline & & OX2E93E577A837AAC & OXF50C2B06C5B100F1D712 & $2,3,5,13,14$ \\
\hline & & OXFDAFFE872B1ECA6 & OX63F0791A5BD92EA49167 & $2,5,7,10,15$ \\
\hline & & OX1B3537B58870F55 & OXA33A411FC4173976088F & $2,3,5,8,9,10,14,15,18$ \\
\hline & & OX1E15EFE0723A1A0 & OXAFF $45320480 \mathrm{C} 32 \mathrm{FE} 05 \mathrm{AD}$ & $2,6,11,12,13,14,15,17$ \\
\hline & & OX137AD462B48819D & OXECFE45F642EA682545D6 & $7,10,17$ \\
\hline & & OX94252897FEBA & OX40B53BED60BA2A4EF7BD & $7,10,11$ \\
\hline & & OX2EEDD3E63910450 & OX33953B8FBB3E5DB89240 & $6,7,17$ \\
\hline & & OX7E51F8E40591536 & OXB9C6CEF795453064BE6 & $9,10,14,15,17$ \\
\hline & & OXD241FF6460C6208 & OX5C725E6A96F9353ЕCCE8 & $2,5,12,14,18$ \\
\hline & & OX895517354CC7691 & OX9099444B625C731D4A9F & 4,13 \\
\hline & & OX18093FF2EA21B89 & OX3627A654C272E098ABA & $8,15,17$ \\
\hline & & OX69219DE1A75C6B5 & OX59FB6A44546208BBA473 & $5,8,9,12,13,15$ \\
\hline & & OX8CA5215750A $80 \mathrm{AE}$ & OXBBFE302CAEC030A95C66 & $2,7,11,18$ \\
\hline & & OX48F6A050FA29FD4 & OXF13127F0DE9243B0AA33 & 10,12 \\
\hline & & OXA869C2C7AE9EEDD & OXFC4D4C6DE13E62F8530E & $2,6,7$ \\
\hline & & OX54D805626D001BA & OXCC3F38A3216216E4DD16 & 10,15 \\
\hline & & OXFF859A14E90D2D3 & OX2AC86B44BBEF20B3A8EB & 3 \\
\hline & & OX92E4EE44F4AA9B0 & OXB2E35370E80D3FA438FE & $5,7,8,9,10,11,12,14$ \\
\hline & & OXC906517717370E8 & OX407C62FEAAD57DE22435 & $6,8,9,15$ \\
\hline & & OX81ABA2F10E414F1 & OXD8DC3E5436B30D553DFF & 13,15 \\
\hline & & OX5E2587E52504105 & OX492770685C260EB94076 & $3,4,5,7,9,11,14$ \\
\hline & & OX66BE1B647D74852 & OXE2B64E025081086192F3 & $2,4,5,7,8,11,13$ \\
\hline & & OXC987FF2679818EA & OXECBC1922F4E $82 \mathrm{FE} 0 \mathrm{E} 298$ & $3,9,14,15,16$ \\
\hline & & OX399995668AA5766 & OXADDE1B907E417C75C073 & 9,18 \\
\hline & & OXA01F4042A39D2AF & OXB42E343741AE2885A4B8 & $8,10,11,15,16$ \\
\hline & & OXEB000173340180D & OX9D4811B44D956C1C4122 & 9,10 \\
\hline & & OX6FEDB601C5D0F7 & OXACF51C7A59AC427CBA18 & $2,4,13,14,16,17,18$ \\
\hline & & OX349068A2D3BE11B & OXF51A7B67A45C5173EDB0 & $2,6,9,10,11,12$ \\
\hline & & OX9904566610C1359 & OX5C921B727602478B4F1F & $2,11,13,18$ \\
\hline & & OX631BFA24A283F98 & OX8BEE5BDF986177DEFCB7 & $2,4,7,8,14,15,17$ \\
\hline & & OX41B6D6E060B45 & OXF4301269A0A373516F83 & $4,6,7,9,15$ \\
\hline & & OX6AE3E77490E6D0B & OXED4B6FCC7E5B1FFAA681 & $2,6,12,15,17$ \\
\hline
\end{tabular}

Continued on next page 
Table 8 - Continued from previous page

\begin{tabular}{|c|c|c|c|c|}
\hline$n$ & Key Variables & Key Constant & IV Constant & $\begin{array}{l}\text { Monomial } \\
\text { Degrees }\end{array}$ \\
\hline \multirow{14}{*}{20} & \multirow{14}{*}{$\begin{array}{c}0,1,9,10,14,19,27,29,41,42 \\
52,55,62,64,68,69,71,75,78,79\end{array}$} & OXF9BB1A903D2B55A & OXBEFF617BF05E74ED8172 & $6,9,11,18$ \\
\hline & & OXADB103911781696 & OX78001622345E7535AF89 & $2,3,10,12,13,14$ \\
\hline & & OXD4BAF7074479E09 & OX3CB7239F46CD1A18C135 & $5,6,13,16,18$ \\
\hline & & OX31C341E7D2823C0 & OX44E6375DA9C323B5EFCF & $15,16,17$ \\
\hline & & OX5A4644E0DCF37F1 & OXAFE71BE0360E0C918B9C & $3,9,13$ \\
\hline & & OXBCF4D6769BCEFB & OX6D344C7B4AC745BC07FC & $10,11,12,18$ \\
\hline & & OXD989E1257E60721 & OX85F9933760D63491E6D & $2,8,13,18$ \\
\hline & & OXFE4EC2B5DF70C 87 & OX7E0D7707ABF24E5811D8 & $4,7,8,10,11,14,17$ \\
\hline & & OX6BB3225B97D099 & OX5BD374B78F0F 10E1F552 & 6 \\
\hline & & OX3666DCC1529A055 & OX998A7DC1F1F94C9CAFB0 & 3,9 \\
\hline & & OXAA07D7C6F262C91 & OX4F821468B1891D2AD371 & $2,3,4,5,7,12$ \\
\hline & & OXCDB760C050E0196 & OX2FDE16E5A5517466E581 & $10,16,18$ \\
\hline & & OX748AA0B4C4431F6 & OX6BB3415153E252D74428 & 9,17 \\
\hline & & OX82641E96DDFE210 & OXA045545ADF754FE 49440 & $4,12,13,16,17$ \\
\hline \multirow{26}{*}{30} & \multirow{26}{*}{$\begin{array}{l}7,15,20,21,22,26,29,30,32,33 \\
34,41,42,49,52,54,55,56,57,59 \\
60,63,64,65,66,72,75,76,78,79\end{array}$} & OX2A3B12E5B3AAA & OX9107625D556D1E48A3B5 & $3,7,13,16,17,18,20,23,25$ \\
\hline & & OX11D3963CFE658 & OXE9F618EC66862A0DEB4E & $9,12,13,14,15,18$ \\
\hline & & $\mathrm{OXF} 8534 \mathrm{CF} 8 \mathrm{~A} 0 \mathrm{C} 4$ & OXCEDD5CCE04F12DF6FA42 & $7,9,10,14,15,18$ \\
\hline & & OXE0D05859F75D & OXDE0D17F6F4A032F 4345A & $2,4,5,8,13,15,16,18$ \\
\hline & & OX1767659F97A78 & OXDB0E189FAA7523B7F38C & $3,5,11,12,13,16,18,19,24$ \\
\hline & & OX3C25D092EFEF9 & OXEE0C5AA49BA676F04E05 & $4,8,10,14,15,23,24$ \\
\hline & & OX358F63BC9862E & OX2B8A12AF7C7513BFB545 & $4,5,8,10,14,15,16,23,25$ \\
\hline & & OXCDCA70B4903D & OX28094F93A84519B6030 & $2,6,12,16,21,24,26$ \\
\hline & & OX186E1140CAE7A & OXE5893222F3CF2AD91C 84 & $3,10,17,21,24$ \\
\hline & & OXF5633C0E0766 & OX3A2161ED1A9A6C545C99 & $6,8,14,16,17,18,23,25$ \\
\hline & & OX3EF1C76CC3786 & OX91441019D7A5F99C0E2 & $5,8,15,16,19,22,26,27$ \\
\hline & & OX1E3305EE66BF7 & OX84052206580263DB7246 & $3,9,12,14,23$ \\
\hline & & OX2BFEF0DB6F4F7 & OX21D64C13071A1E0AA4DF & $10,14,24,28$ \\
\hline & & OX22BE07DCB8255 & OX14B48826D4E3EE8AA4A & $10,11,13,14,17,22,24,25$ \\
\hline & & OX2C53E5CA904F8 & OX4CF8318FB91A7BD1C2D0 & $4,5,8,14,17,18,19,22,24,27$ \\
\hline & & OX93726691E2D0 & $\mathrm{OX} 2 \mathrm{C} 4 \mathrm{C} 389 \mathrm{C} 765606937 \mathrm{AF} 4$ & $6,10,15,18,19,13,16$ \\
\hline & & OX3ED1D244BD2B1 & OXBB5B758E8FB029E57666 & $2,6,7,8,15,23,25$ \\
\hline & & OX2DF6C79AE5433 & OX920D16223BEE4EB5822E & $3,4,8,9,12,13,20$ \\
\hline & & OX378C02C3FDF2B & OX77681A2286592408308D & $3,10,11,13,15,22,23,26,28$ \\
\hline & & OX3B97E24D24147 & OX694F280DCB2B108F1385 & $2,9,11,12,24,28$ \\
\hline & & OXC7294829B50A & OXF303799BF930108F4B0F & $4,8,11,15,16$ \\
\hline & & OX378763FE6C96 & OX2FBC5FB87C8125734B6E & $2,3,6,7,8,12,16,18,23,25,28$ \\
\hline & & OX3BECF5CC75818 & OXC3B33304C9FD300B28F3 & $5,12,14,17,19,21,22,23,24$ \\
\hline & & OX3049A0E2FB512 & OX50986952B94273E8F099 & $9,13,16,24,26$ \\
\hline & & OX313C07B28B127 & OX30005763E511714B24C0 & $10,11,17,18,25,26$ \\
\hline & & OX613242AFA99E & OX74996C308B57426EC1FF & $4,5,8,11,12,13,15, \underset{27}{17}, 19,21,22,24$ \\
\hline
\end{tabular}

Continued on next page 
Table 8 - Continued from previous page

\begin{tabular}{|c|c|c|c|c|}
\hline$n$ & Key Variables & Key Constant & IV Constant & $\begin{array}{c}\text { Monomial } \\
\text { Degrees }\end{array}$ \\
\hline \hline \multirow{2}{*}{30} & $7,15,20,21,22,26,29,30,32,33$, & OX5AE80FC1F4DB & OXD5776A122F7F7B0049B1 & $3,10,15,21,23,24$ \\
\cline { 3 - 5 } & $34,41,42,49,52,54,55,56,57,59$, & OX191766116C74F & OX1E8B7D71045E0F56A4EA & $4,13,17,18,24,25$ \\
\cline { 3 - 5 } & $60,63,64,65,66,72,75,76,78,79$ & & &
\end{tabular}

